\title{
Associative Memories Applied to Image Categorization
}

\author{
Roberto A. Vázquez and Humberto Sossa \\ Centro de Investigación en Computación - IPN \\ Av. Juan de Dios Batíz, esquina con Miguel Othón de Mendizábal \\ Ciudad de México, 07738, México. \\ ravemaipn.mx, hsossa@cic.ipn.mx
}

\begin{abstract}
In this paper we describe how associative memories can be applied to categorize images. If we present to an associative memory (AM) an image we would expect that the AM would respond with something that describes the content of the image; for example, if the image contains a tiger we would expect that the AM would respond with the word "tiger". In order to achieve this goal, we first chose a set of images. Each image is next associated to the word that better describes the content of the image. With this information an AM is trained as in [10]. We then use the AM to categorize instances of images with the same content even if these images are distorted by some kind of noise. The accuracy of the proposal is tested using a set of images containing different species of flowers and animals.
\end{abstract}

\section{Introduction}

Research on associative memories (AMS) has been carried out during the last 40 years. AMS can be seen as a particular kind of neural networks. AMS are a mathematical device specially designed to recall output patterns in terms of input patterns which can be contaminated by some kind of noise, see for example [1-9]. Some of these AMS have several constraints that limit their applicability in the solution real life problems. Most common applications of AMS are as filters [5-7]; in this case the AM is fed with a signal (an image, for example), possibly altered by noise; at the output the original image (without noise) should be obtained. However, in order to achieve the best performance the input patterns have to satisfy some restrictions; for example in [5-6] the input pattern can only be distorted by additive or subtractive noise, but not both. These conditions limit their applicability in real life problems. In [7] the patterns can be contaminated with both types of noise. Another application of AMS is as classification tools [8-9], however they were only tested in the classification of simple objects. Recently in [10] it was introduced a new associative model which has demonstrated to be useful for both applications (as a filter and as a classification tool); due to its robustness, the model has been recently applied to retrieve images from a database [11]. In this paper, we adopt the same model to solve the problem of image categorization.

Most of the information on the web is disposed as images; however this information is not well organized. In order to organize those millions of images it is necessary categorize them in some manner. In [12], for example, the authors use objective 
semantic cues for the semantic indexing and quering of images. In this paper we describe how images can be categorized using the AM model recently introduced in [10]. If we feed an AM with a picture we expect that it would respond with something indicating the content of the picture. If, for example the picture contains a tiger, we would expect that the AM would respond with the word "tiger". With this goal in mind, we first select a set of images; we then manually associate these images with a description of the images. With these associations we finally train the corresponding AM. To test the proposal we use a set of images containing different species of flowers and animals; we expect to categorize instances of these images even if they appear distorted by noise.

The remaining of the paper is organized as follows. In section 2, we describe the adopted AM model. In section 3, we describe how the proposal is implemented, while in section 4 , we present the obtained experimental results. In section 5, we finally conclude and give some directions for further research.

\section{Associative Model}

An association between input pattern $\mathbf{x}$ and output pattern $\mathbf{y}$ is denoted as $\left(\mathbf{x}^{k}, \mathbf{y}^{k}\right)$, where $k$ is the corresponding association. The associative memory $\mathbf{M}$ is represented by a matrix whose component $m_{i j}$ can be seen as the synapses neuron $i$ and neuron $j . \mathbf{M}$ is generated from a finite a priori set of know associations, known as the fundamental set of association and is represented as: $\left\{\left(\mathbf{x}^{k}, \mathbf{y}^{k}\right) \mid k=1,2, \ldots, p\right\}$ where $p$ is the number of associations. If $\mathbf{x}^{k}=\mathbf{y}^{k} \forall k \in\{1,2, \ldots p\}$ then $\mathbf{M}$ is auto-associative, otherwise it is hetero-associative. A distorted version of a pattern $\mathbf{x}$ to be restored will be denoted as $\widetilde{\mathbf{x}}$. If an associative memory $\mathbf{M}$ is fed with a distorted version of $\mathbf{x}^{k}$ and the output obtained is exactly $\mathbf{y}^{k}$, we say that recalling is perfect.

\subsection{Building and Testing the Associative Memory}

Two main phases are used to build and test the AM model.

\section{Training Phase:}

1. For each couple $\left\{\left(\mathbf{x}^{k}, \mathbf{y}^{k}\right) \mid k=1,2, \ldots, p\right\}$ build matrix $\mathbf{y} \diamond_{\mathrm{A}} \mathbf{x}^{t}$ as:

$$
\mathbf{y} \diamond_{\mathrm{A}} \mathbf{x}^{t}=\left(\begin{array}{cccc}
\mathrm{A}\left(y_{1}, x_{1}\right) & \mathrm{A}\left(y_{1}, x_{2}\right) & \cdots & \mathrm{A}\left(y_{1}, x_{n}\right) \\
\mathrm{A}\left(y_{2}, x_{1}\right) & \mathrm{A}\left(y_{2}, x_{2}\right) & \cdots & \mathrm{A}\left(y_{2}, x_{n}\right) \\
\vdots & \vdots & \ddots & \vdots \\
\mathrm{A}\left(y_{m}, x_{1}\right) & \mathrm{A}\left(y_{m}, x_{2}\right) & \cdots & \mathrm{A}\left(y_{m}, x_{n}\right)
\end{array}\right)_{m \times n}
$$

with $\mathrm{A}(x, y)=x-y$. 
2. Apply the median operator to the matrix obtained in step1 to get matrix $\mathbf{M}$.

$$
m_{i j}=\underset{k=1}{p} \operatorname{med} \mathrm{A}\left(y_{i}^{k}, x_{j}^{k}\right)
$$

\section{Recalling Phase:}

A pattern $\mathbf{x}^{k}$ is presented to the memory $\mathbf{M}$ and the following operation is done to recall $\mathbf{y}^{k}$ :

$$
\left(\mathbf{M} \diamond_{\mathrm{B}} \widetilde{\mathbf{x}}^{k}\right)_{i}=\operatorname{mid}_{j=1}^{n} \mathrm{~B}\left(m_{i j}, \widetilde{x}_{j}^{k}\right)
$$

In this case $\mathrm{B}(x, y)=x+y$ and operator mid is defined as $\operatorname{mid} \mathbf{x}=x_{(n+1) / 2}$.

\subsection{A Dynamic Associate Memory}

Humans, in general, do not have problems to recall patterns even in the presence of noise. Before an input pattern is learned or processed by the brain, it is hypothesized that it is transformed and codified by the brain. This process can be simulated using the algorithm described in [8]:

Procedure 1. Transform the fundamental set of associations into codified patterns and de-codifier patterns:

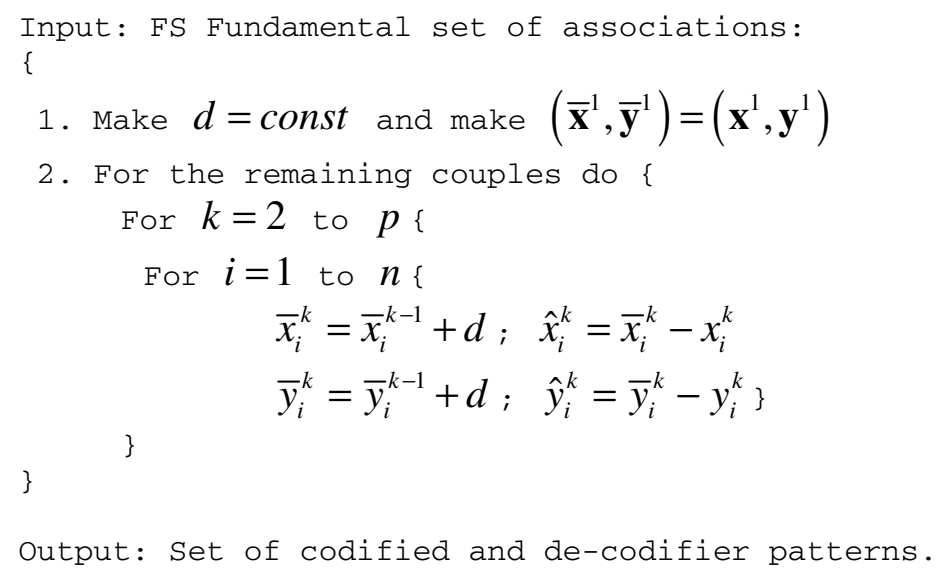

This procedure allows computing codified patterns from input and output patterns denoted by $\overline{\mathbf{x}}$ and $\overline{\mathbf{y}}$, respectively. On the other hand $\hat{\mathbf{x}}$ and $\hat{\mathbf{y}}$ are the de-codifier patterns. In addition a simplified version of $\mathbf{x}^{k}$ denoted by $s_{k}$ is obtained using:

$$
s_{k}=s\left(\mathbf{x}^{k}\right)=\operatorname{mid} \mathbf{x}^{k}
$$


When the brain is stimulated by an input pattern, some regions of the brain are stimulated by its information, also are the synapses belonging to that region. We call these regions active regions and are computed as follow:

$$
\operatorname{ar}=r(\mathbf{x})=\arg \left(\widehat{\wedge}_{i=1}^{p}\left|s(\mathbf{x})-s_{i}\right|\right)
$$

Principal synapses (kernel of the AM) are located in the middle column of matrix $\mathbf{M}$ and is denoted by $\mathbf{K}_{\mathbf{M}}=\underset{i=1}{m} w_{i}$. The synapses that belong to $\mathbf{K}_{\mathbf{M}}$ are modified as a response to the input pattern. This pattern stimulates some regions, interacts with these active regions and then, according to those interactions modifies the synapses. Adjusting factor is denoted by $\Delta w$ and is given as:

$$
\Delta w=\Delta(\mathbf{x})=s\left(\overline{\mathbf{x}}^{r}\right)-s(\mathbf{x})
$$

where $r$ is the index of the active region.

Finally synapses belonging to $\mathbf{K}_{\mathbf{M}}$ are updated as:

$$
\mathbf{K}_{\mathbf{M}}=\mathbf{K}_{\mathbf{M}} \oplus\left(\Delta w_{\text {new }}-\Delta w_{\text {old }}\right)
$$

where operator $\oplus$ is defined as $\mathbf{x} \oplus d=x_{i}+d \forall i=1, \ldots, m$.

Using this dynamic approach an AM can be built using the following procedure:

1. Transform the fundamental set of association into codified and de-codifier patterns using procedure 1.

2. Compute simplified versions of input patterns using equation 3.

3. Build matrix $\mathbf{M}$ in terms of codified patters: apply step 1 and 2 of the training procedure described at the beginning of section 2 .

Given a pattern $\mathbf{x}^{k}$ or a distorted version of it $\tilde{\mathbf{x}}^{k}$, pattern $\mathbf{y}^{k}$ can be recovered as follows:

1. Obtain index of active region $a r$ by means of equation 4 .

2. Transform $\mathbf{x}^{k}$ using de-codifier pattern $\hat{\mathbf{x}}^{a r}$ as: $\widehat{\mathbf{x}}^{k}=\mathbf{x}^{k}+\hat{\mathbf{x}}^{a r}$.

3. Compute adjusting factor $\Delta w=\Delta(\hat{\mathbf{x}})$ by using equation 5 .

4. Modify synapses of associative memory $\mathbf{M}$ that belong to $\mathbf{K}_{\mathbf{M}}$ by means of equation 6 . 
5. Apply step 1 of the recalling phase described in section 2 .

6. Obtain $\mathbf{y}^{k}$ by transforming $\hat{\mathbf{y}}^{k}$ by using de-codifier pattern $\hat{\mathbf{y}}^{a r}$ as : $\mathbf{y}^{k}=\widehat{\mathbf{y}}^{k}-\hat{\mathbf{y}}^{a r}$.

\section{Categorizing Images}

Image categorization is not trivial when pictures are taken from real life situations. This implies that categorization must be invariant to several image transformations such as translations, rotations, scale changes, illumination changes, orientation changes, noise, and so on.

A first step to solve this problem, we provide a solution when the concerned images are distorted only by mixed noise. For this we use the associative model described in section 2. Firstly, we select a set of images. We then associate these images with describing words. The images and the describing words are our set of associations, with $\mathbf{x}^{k}$ is the $k$-image and $\mathbf{y}^{k}$ the $k$-describing word. With this set of associations we next proceed to build the corresponding associative memory as explained in section 2.1.

Either for learning or recall, each color image $f_{k}(i, j)$ is converted to a vector by means of standard scanning method. If image $f_{k}(i, j)$ is of size $M \times N$ pixels, then its corresponding vector is $\mathbf{x}^{k}=\left[\begin{array}{llll}x_{1}^{k} & x_{2}^{k} & \ldots & x_{M \times N}^{k}\end{array}\right]^{T}$.

The elements $y_{r}^{k}, r=1, \ldots, R$ of vector $\mathbf{y}^{k}$ correspond to the ASCII codes of the letters of each describing word. $R$ is the number of letters of a given word.

Something important with this proposal is that we can control the degree of detail of categorization going from something general to something particular. Let us suppose, for example, that the set of selected images contain just animals, and that then we can categorize these animals into oviparous or viviparous. If we want more detail, we can categorize oviparous animal in reptiles and birds, and so on.

\section{Experimental Results}

In this section, we test the accuracy of the proposal with a set of 40 images containing flowers and animals as shown Figure 1.

Three experiments where performed in order to determine the accuracy of the proposal. We use different description levels. In the first experiment we categorized the set images into classes: flowers or animals. In second experiment we went more in detail and split flowers and animals into different families of species, eleven families for flowers and six families for animals; seventeen classes in total. In the last experiment, we categorized each flower and animal into its particular name; here we categorized the images into forty classes. 


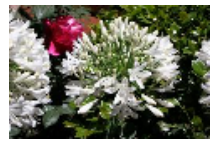

Agapanthus

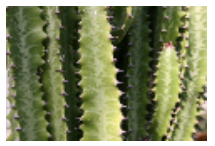

Cactus

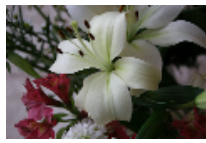

White flower

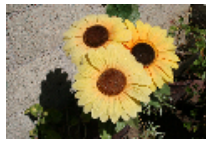

Sunflower

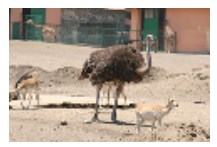

Ostrich

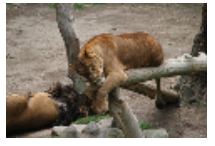

Lion

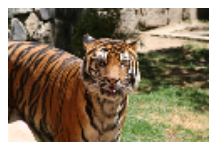

Tiger

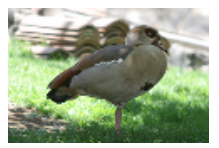

Goose

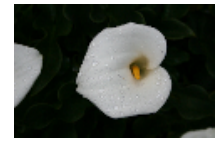

White calla lily

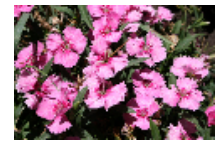

Dianthus

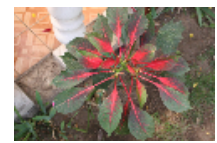

Green-red flower

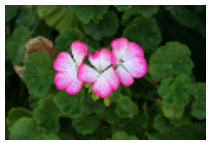

Cranesbills

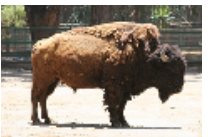

Bison

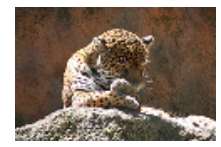

Leopard

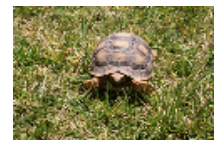

Turtle

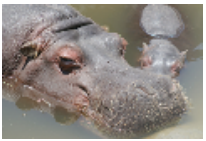

Hippopotamus

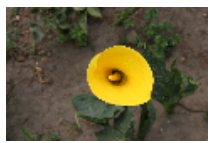

Yellow calla lily

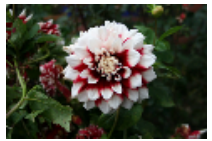

Dahlia

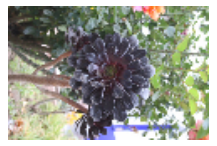

Black flower

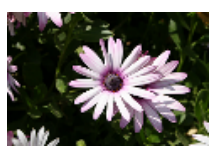

Daisy

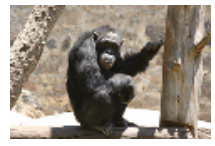

Chimpanzee

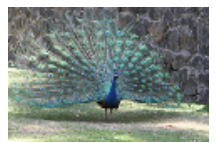

Peacock

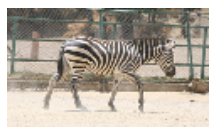

Zebra

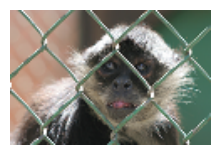

Spider monkey

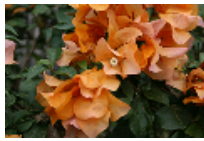

Yellow bougainvillea

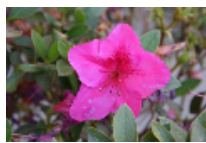

Purple flower

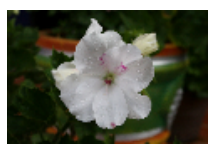

White geranium

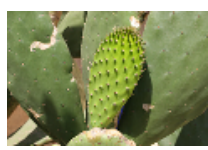

Nopals

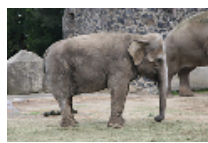

Elephant

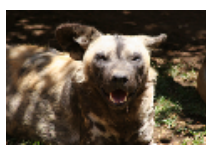

Wild dog

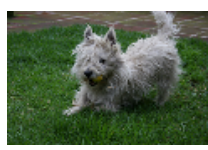

Domestic dog

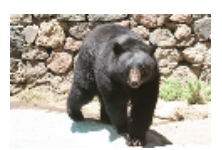

Bear

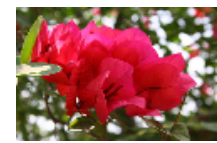

Purple bougainvillea

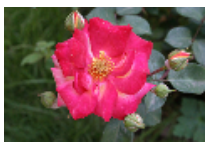

Red flower

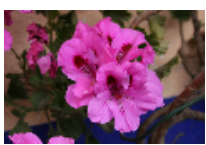

Pink geranium

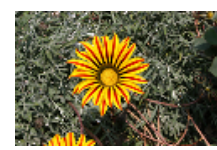

Gazania

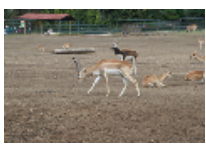

Impala

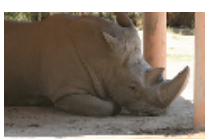

Rhinoceros

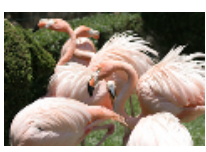

Flamingo

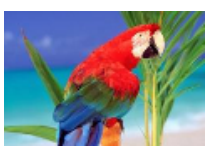

Macaw

Fig. 1. Set of images of flowers and animals used in the experiments

In the first experiment we associated whole flowers with the description word "flowers" and complete animals with the description word "animals". These associations allow us to categorize the images into two classes; this is a description of level 1, see Figure 2. 


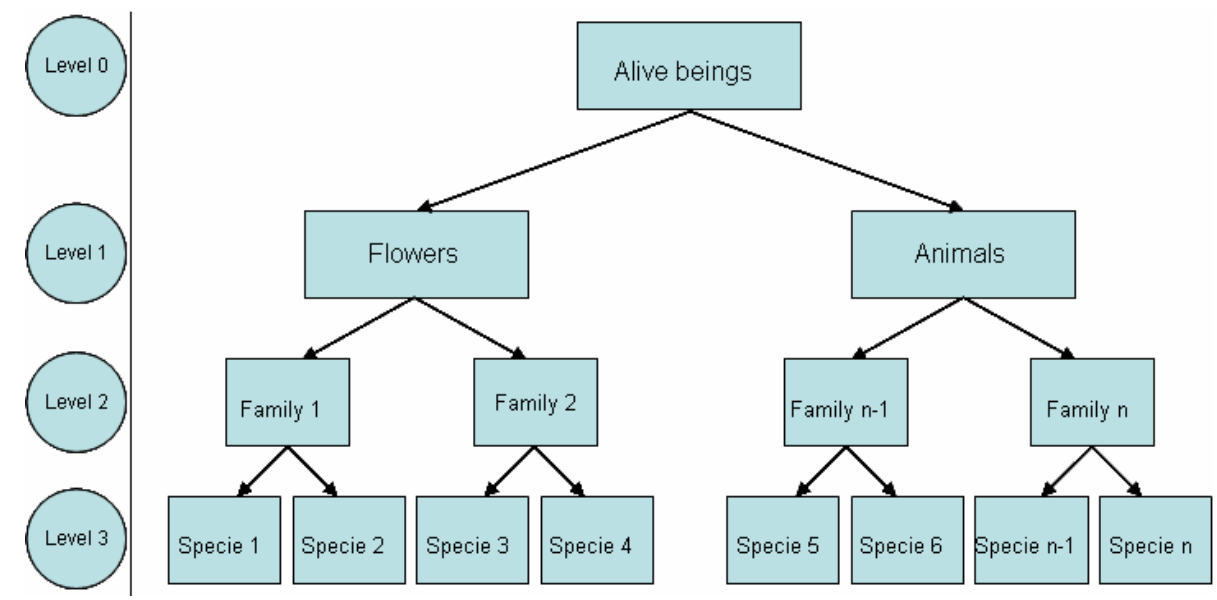

Fig. 2. Description levels used to train the associative memory in the three different experiments

For the second experiment we decided to categorize the images into different families, see Table 1 . In this case we associated the flower and the animals belonging to a specific family with the name of the family.

Table 1. Different species of flowers and animals associated to a specific family. This implies that if the associative memory is fed with an image of a lion, leopard or tiger, the associative memory will respond with the word "felines" and so on.

\begin{tabular}{|l|l|}
\hline Family (description word) & Species \\
\hline Agapanthus & Agapanthus \\
\hline Calla lily & White calla lily, yellow call lily \\
\hline Bougainvillea & Yellow bougainvillea, purple bougainvillea \\
\hline Cactaceae & Cactus, nopals \\
\hline Dianthus & Dianthus \\
\hline Dahlia & Dahlia \\
\hline Other flowers & Purple flower, red flower, white flower, green-red flower, black flower \\
\hline Geranium & White geranium, pink geranium, cranesbills \\
\hline Daisy & Daisy \\
\hline Gazania & Gazania \\
\hline SunFlower & Sunflower \\
\hline Birds & Ostrich, peacock, flamingo, goose, macaw \\
\hline Primates & Chimpanzee, spider monkey \\
\hline Felines & Lion, leopard, tiger \\
\hline Dogs & Wild dog, domestic dogs \\
\hline Big animals & Bison, elephant, hippopotamus, rhinoceros, bear \\
\hline Small animas & Impala, turtle, zebra \\
\hline
\end{tabular}

For the last experiment we associated each image with the name of the specie appearing on the image, see Table 2. 
Table 2. Each species is associated with the name of the specie. This implies that if the associative memory is fed with the image of the tiger, the associative memory will respond with the word "tiger" and so on.

\begin{tabular}{|l|l|l|l|}
\hline Description word & Specie & Description word & Specie \\
\hline Agapanthus & Agapanthus & White flower & White flower \\
\hline White calla lily & White calla lily & Green-red flower & Green-red flower \\
\hline Yellow calla lily & Yellow calla lily & Black flower & Black flower \\
\hline Yellow bougainvillea & Yellow bougainvillea & White geranium & White geranium \\
\hline Purple bougainvilie & Purple bougainvilie & Pink geranium & Pink geranium \\
\hline Cactus & Cactus & Sunflower & Sunflower \\
\hline Dianthus & Dianthus & Cranesbills & Cranesbills \\
\hline Dahlia & Dahlia & Daisy & Daisy \\
\hline Purple flower & Purple flower & Nopal & Nopal \\
\hline Red flower & Red flower & Gazania & Gazania \\
\hline Ostrich & Ostrich & Tiger & Tiger \\
\hline Bison & Bison & Turtle & Turtle \\
\hline Chimpanzee & Chimpanzee & Zebra & Zebra \\
\hline Elephant & Elephant & Domestic dog & Domestic dog \\
\hline Impala & Impala & Flamingo & Flamingo \\
\hline Lion & Lion & Goose & Goose \\
\hline Leopard & Leopard & Hippopotamus & Hippopotamus \\
\hline Peacock & Peacock & Spider monkey & Spider monkey \\
\hline Wild dog & Wild dog & Bear & Bear \\
\hline Rhinoceros & Rhinoceros & Macaw & Macaw \\
\hline
\end{tabular}

Once the associative memory in each experiment was trained we manually contaminated the images with noise and then we fed the associative memories with the contaminated image in order to obtain its categorization.

As you can appreciate in Figure 3, whole images were perfectly categorized in the three experiments. These results support the accuracy of the proposal.

\section{Conclusion and Ongoing Research}

In this paper we have described how an associative memory can be used for categorizing images containing complex objects as flowers and animals. Although only the proposal was tested with images manually modified, the results were highly acceptable.

It is important to mention that this is the first time that an associative memory is used to categorize images, and the most important, complex images with flowers and animals.

In addition, we have categorized images in three different description levels. We performed three experiments going from a general description to a particular description. These descriptions can also be arranged into a hierarchical tree of descriptions. On the other hand we have demonstrated the robustness of the associative model categorizing images in more than twenty classes.

The associative model used is robust under some affine transformations but only when the images contain plastic objects; however with complex images the associative model could provide a low accuracy. 


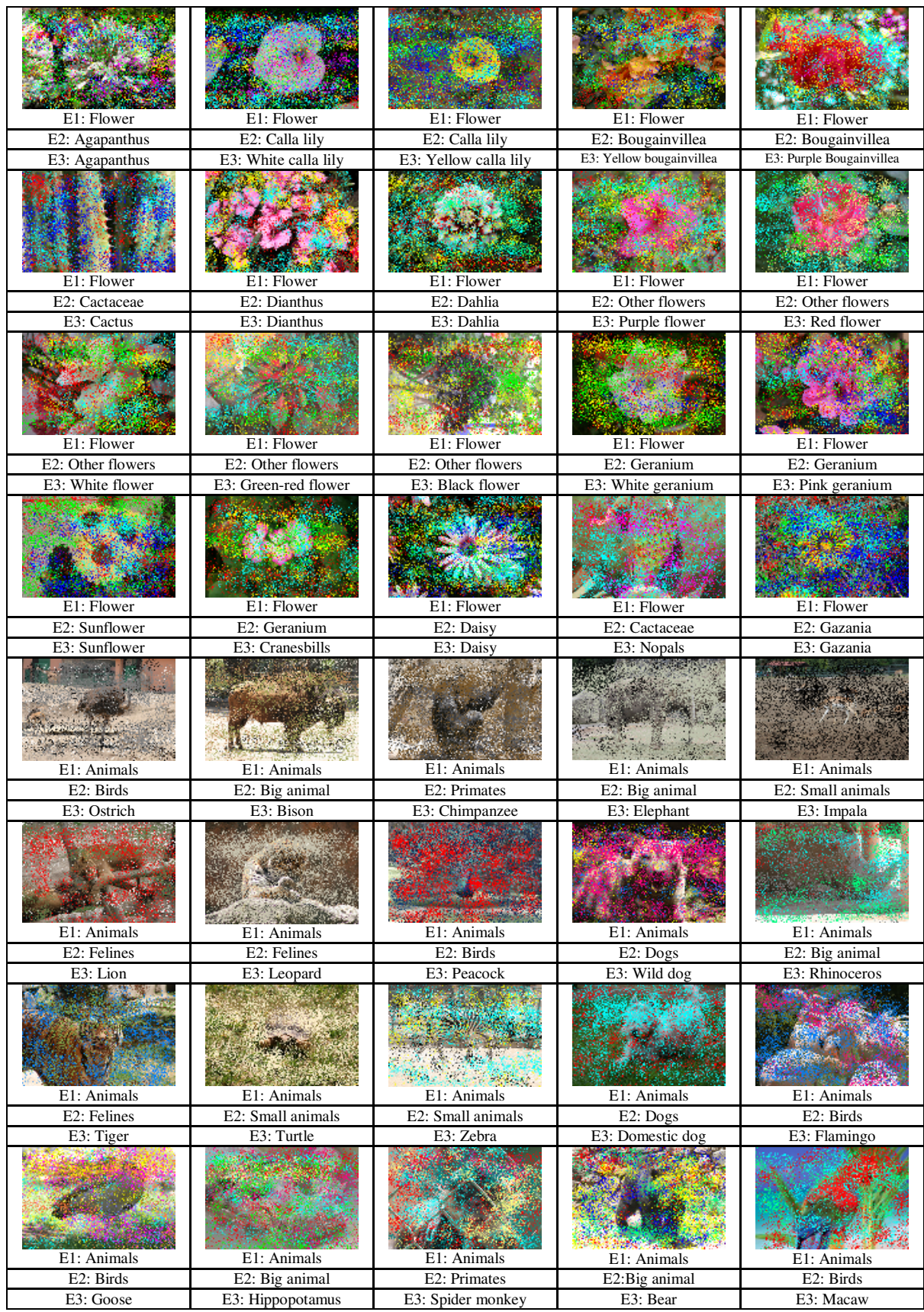

Fig. 3. Results obtained in the experiments using altered images. E1:xx represents the description word obtained by feeding the memory with the image in experiment 1 . The same occurs for E2 and E3. 
Nowadays we are facing more complex problems, for example when the images present illumination changes and some image transformations, such as translations, rotations, scale changes, and so on. By solving all of these problems and by combining the solution with a hierarchical arrangement of associative memories it might be possible to develop a powerful image retrieval system.

Acknowledgements. This research was economically supported by SIP-IPN under grants 20050156 and 20060517 and SEP-CONACYT by means of grant SEP-2004C01-46806/1215.

\section{References}

1. K. Steinbuch (1961). Die Lernmatrix. Kybernetik, 1(1):26-45.

2. J. A. Anderson (1972). A simple neural network generating an interactive memory. Mathematical Biosciences, 14:197-220.

3. T. Kohonen (1972). Correlation matrix memories. IEEE Trans. on Computers, 21(4): 353-359.

4. J. J. Hopfield (1982). Neural networks and physical systems with emergent collective computational abilities. Proceedings of the National Academy of Sciences, 79: 2554-2558, 1982.

5. P. Sussner (2003). Generalizing operations of binary auto-associative morphological memories using fuzzy set theory. Journal of mathematical Imaging and Vision, 19(2): 81-93.

6. G. X. Ritter, G. Urcid, L. Iancu (2003). Reconstruction of patterns from noisy inputs using morphological associative memories. Journal of mathematical Imaging and Vision, 19(2):95-111.

7. H. Sossa and R. Barron (2003). New associative model for pattern recall in the presence of mixed noise. In Proceedings of the fithh IASTED International Conference on Signal and Image Processing, SIP2003. Acta Press 399:485-490.

8. H. Sossa, R. Barrón, R. A. Vázquez (2004). Transforming Fundamental set of Patterns to a Canonical Form to Improve Pattern Recall. LNAI 3315:687-696. Springer Verlag.

9. H. Sossa, R. Barrón, R. A. Vázquez (2004). New associative memories for recall realvalued patterns. LNCS 3287:195-202. Sprinegr Verlag.

10. R. A. Vázquez, H. Sossa and R. Barrón (2006). Enhanced associative memory model for pattern restoration. To be submitted.

11. R. A. Vázquez and H. Sossa (2006). Associative memories applied to image retrieval. Submitted to MICAI 2006.

12. A, Mojsilović, J. Gomes and B. Rogowitz (2004). Semantic-Friendly Indexing and Quering of Images Based on the Extraction of the Objective Semantic Cues. International Journal of Computer Vision, Special Issue on Content-Based Image Retrieval, 56(1-2):79-107. 